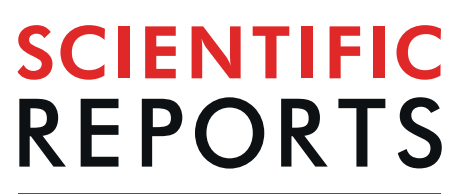

natureresearch

\title{
OPEN A multiplex qPCR approach for detection of pfhrp2 and pfhrp3 gene deletions in multiple strain infections of Plasmodium falciparum
}

Received: 13 May 2019

Accepted: 24 August 2019

Published online: 11 September 2019

\author{
Tobias Schindler $\mathbb{D}^{1,2}$, Anna C. Deal ${ }^{1,2}$, Martina Fink ${ }^{1,2}$, Etienne Guirou $\mathbb{D}^{1,2}$, Kara A. Moser ${ }^{3}$, \\ Solomon M. Mwakasungula ${ }^{4}$, Michael G. Mihayo ${ }^{4}$, Said A. Jongo ${ }^{4}$, Prosper P. Chaki ${ }^{4}$, \\ Salim Abdulla ${ }^{4}$, Paulo C. Manrique Valverde ${ }^{5}$, Katherine Torres ${ }^{5}$, Jose R. Bijeri ${ }^{6}$, Joana C. Silva ${ }^{5}$, \\ Stephen L. Hoffman ${ }^{7}$, Dionicia Gamboa ${ }^{5}$, Marcel Tanner ${ }^{1,2}$ \& Claudia Daubenberger ${ }^{1,2}$
}

The rapid and accurate diagnosis of Plasmodium falciparum malaria infection is an essential factor in malaria control. Currently, malaria diagnosis in the field depends heavily on using rapid diagnostic tests (RDTs) many of which detect circulating parasite-derived histidine-rich protein 2 antigen (PfHRP2) in capillary blood. $P$. falciparum strains lacking PfHRP2, due to $p$ fhrp2 gene deletions, are an emerging threat to malaria control programs. The novel assay described here, named qHRP2/3-del, is well suited for high-throughput screening of $P$. falciparum isolates to identify these gene deletions. The qHRP2/3del assay identified $p f h r p 2$ and $p f h r p 3$ deletion status correctly in $93.4 \%$ of samples with parasitemia levels higher than 5 parasites/ $\mu \mathrm{L}$ when compared to nested PCR. The qHRP2/3-del assay can correctly identify $p f h r p 2$ and $p f h r p 3$ gene deletions in multiple strain co-infections, particularly prevalent in SubSaharan countries. Deployment of this qHRP2/3-del assay will provide rapid insight into the prevalence and potential spread of $P$. falciparum isolates that escape surveillance by RDTs.

Malaria is an infectious disease with an estimated 219 million cases globally and was responsible for 435'000 deaths in 2017. More than $90 \%$ of these malaria cases and deaths occurred in sub-Saharan Africa with Plasmodium falciparum as the most pathogenic malaria parasite species, accounting for the vast majority of clinical malaria cases ${ }^{1}$.

Advances have been made in malaria control which have contributed to the decline in malaria prevalence observed worldwide with improved diagnostic tests and better access to malaria treatment contributing significantly to this development ${ }^{1}$. The rapid and accurate diagnosis and treatment of malaria cases is an essential factor in the control of malaria. Rapid diagnostic tests (RDTs) are becoming the most widely used method to diagnose malaria infections in the field with 245 million RDTs distributed worldwide in $2017^{1}$. In sub-Saharan Africa an estimated $75 \%$ of malaria tests conducted in 2017 were based on RDTs ${ }^{1}$. Malaria RDTs are based on an immuno-chromatographic assay using a lateral-flow device which allows the detection of malaria antigens in usually 5 to $15 \mu \mathrm{L}$ of capillary blood ${ }^{2}$. RDTs provide results within 20 minutes and can be employed by inexperienced health workers operating in resource-limited settings ${ }^{3}$. RDTs recognizing circulating histidine-rich protein 2 (PfHRP2) for sensitive and specific detection of $P$. falciparum make up more than $90 \%$ of RDTs currently in

${ }^{1}$ Department of Medical Parasitology and Infection Biology, Swiss Tropical and Public Health Institute, Basel, Switzerland. ${ }^{2}$ University of Basel, Basel, Switzerland. ${ }^{3}$ Institute for Genome Sciences, University of Maryland School of Medicine, Baltimore, Maryland, USA. 'Bagamoyo Research and Training Centre, Ifakara Health Institute, Bagamoyo, United Republic of Tanzania. ${ }^{5}$ Laboratorios de Investigacion y Desarrollo, Facultad de Ciencias y Filosofia \& Instituto de Medicina Tropical, Alexander von Humboldt Universidad Peruana Cayetano Heredia, Lima, Peru. ${ }^{6}$ Equatorial Guinea Malaria Vaccine Initiative, Malabo, Equatorial Guinea. ${ }^{7}$ Sanaria Inc., Rockville, Maryland, USA. Correspondence and requests for materials should be addressed to T.S. (email: tobias.schindler@unibas.ch) or C.D. (email: claudia.daubenberger@swisstph.ch) 


\begin{tabular}{|c|c|c|c|c|c|}
\hline Target gene & Size & Oligo name & Oligo sequence $\left[5^{\prime}\right.$ to $\left.3^{\prime}\right]$ & Fluorophores & Conc. in $5 \times$ PrimerMix $^{\mathrm{a}}$ \\
\hline \multirow{3}{*}{$\begin{array}{l}\text { pfrnr2e2 } \\
\text { (PF3D7_1015800) }\end{array}$} & \multirow{3}{*}{$107 \mathrm{bp}$} & IC-PfRNR2E2 fwd & AGTATCCAAAACACTATAATTCCAAGTAC & - & $1.5 \mu \mathrm{M}$ \\
\hline & & IC- PfRNR2E2 rev & ATTTTCTCСTTTCTTAACAGTTTCTTCC & - & $1.5 \mu \mathrm{M}$ \\
\hline & & IC-PfRNR2E2 Cy5 & CCTTTTAGTTGGCCCGAATTTACAA & Cy5-BHQ2 & $1.125 \mu \mathrm{M}$ \\
\hline \multirow{3}{*}{$\begin{array}{l}\text { pfhrp2 } \\
\text { (PF3D7_0831800) }\end{array}$} & \multirow{3}{*}{$286 \mathrm{bp}$} & PfHRP2 fwd ${ }^{b}$ & GTATTATCCGCTGCCGTTTTTGCC & - & $1.5 \mu \mathrm{M}$ \\
\hline & & PfHRP2 $\mathrm{rev}^{\mathrm{b}}$ & TCTACATGTGCTTGAGTTTCG & - & $1.5 \mu \mathrm{M}$ \\
\hline & & PfHRP2 TxRd & TTCCGCATTTAATAATAACTTGTGTAGC & TexasRed-BHQ2 & $0.375 \mu \mathrm{M}$ \\
\hline \multirow{3}{*}{$\begin{array}{l}p f h r p 3 \\
\text { (PF3D7_1372200) }\end{array}$} & \multirow{3}{*}{$289 \mathrm{bp}$} & PfHRP3 fwd & ATATTATCCGCTGCCGTTTTTGCT & - & $1.5 \mu \mathrm{M}$ \\
\hline & & PfHRP3 rev & CCTGCATGTGCTTGACTTTCGT & - & $1.5 \mu \mathrm{M}$ \\
\hline & & \begin{tabular}{|l|} 
PfHRP3 YY \\
\end{tabular} & CTCCGAATTTAACAATAACTTGTTTAGC & YakimaYellow-BHQ2 & $0.75 \mu \mathrm{M}$ \\
\hline
\end{tabular}

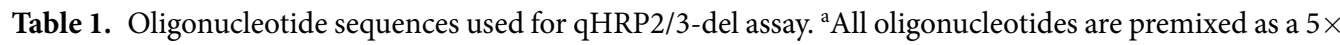
primer mix. ${ }^{b}$ Oligonucleotide sequences obtained from Abdallah et al. ${ }^{32}$.

use $^{4}$. The relatively high abundance and stability of PfHRP2 in the blood of infected patients and expression by $P$. falciparum during the erythrocytic stage makes this antigen a valuable biomarker for malaria infection ${ }^{5}$. PfHRP3, a protein also expressed by $P$. falciparum with high level of structural similarity to PfHRP2, might be also recognized by some of the monoclonal antibodies used in the RDTs ${ }^{6}$. RDTs are critical diagnostic tools for identifying symptomatic malaria infections; however, due to the reduced performance in infections with low parasite density, its use for the diagnosis of malaria infection in asymptomatic individuals is rather limited ${ }^{7}$.

Recent studies report on reduced diagnostic performance of PfHRP2-based RDTs which were attributed to genetic diversity of the $p$ fhrp $2 / 3$ genes ${ }^{6}$, differences in expression level of PfHRP $2 / 3$ antigen in parasite field strains ${ }^{8}$ or isolates lacking $p f h r p 2$ and/or $p f h r p 3$ genes $^{9}$. P. falciparum isolates lacking $p f h r p 2$ and/or $p f h r p 3$ genes are found around the world, with different proportions of the circulating $P$. falciparum population affected. The regions with the highest proportions of $P$. falciparum strains carrying $p$ fhrp 2 deletions are South America and sub-Saharan Africa ${ }^{10}$. Since malaria control programmes depend on reliable diagnosis of malaria cases using RDTs, parasites lacking $p$ fhrp $2 / 3$ genes pose a threat to malaria control and local elimination efforts ${ }^{11}$.

The presence or absence of $p$ fhrp $2 / 3$ genes is usually determined by amplifying these genes by polymerase chain reaction (PCR). Several different (nested) PCR protocols have been published and a deletion is reported if there is no amplification of the $p f h r p 2 / 3$ genes in the presence of an amplification in at least two P. falciparum single copy genes ${ }^{12}$. The conventional nested PCR methods are time consuming, requiring separate reactions for each target gene amplification as well as gel electrophoresis for visualization of the PCR products. Additionally, there are methodological issues related to this approach which assumes identical PCR performance of the $p f h r p 2 / 3$ and the reference genes. Particularly at lower parasitemia levels with a small number of DNA target molecules present, unavoidable stochastic effects can play a major role and might lead to false reporting of $p f h r p 2 / 3$ deletions. Furthermore, none of the published methods detecting $p$ fhrp $2 / 3$ deletions can identify "masked" deletions in multiple strain infections with only one out of several $P$. falciparum strains carrying a $p$ fhrp 2 and/or $p f h r p 3$ gene deletion ${ }^{13}$. These limitations of recommended molecular monitoring methods could result in an underestimation of the prevalence of $P$. falciparum strains with $p f h r p 2 / 3$ deletions, especially in regions with high proportions of multiple strain co-infections.

This paper presents a novel, quantitative PCR-based method for detecting $p f h r p 2$ and $p f h r p 3$ gene deletions suitable for high throughput screening of $P$. falciparum isolates. The qHRP2/3-del (quantitative detection of $p f \underline{h r p 2}$ and $p f \underline{h r p 3}$ deletion) assay was developed as a multiplex assay, with the ability to amplify individually and specifically the $p f h r p 2$ and $p f h r p 3$ genes together with a single copy gene, the $P$. falciparum ribonucleotide reductase R2_e2 (pfrnr2e2) $)^{14}$, as an internal reference. The quantitative nature of the qHRP2/3-del assay provides the basis for estimating the proportions of $P$. falciparum strains carrying $p f h r p 2$ and $p f h r p 3$ deletions in regions with multi-clonal malaria infections.

\section{Results}

Design and evaluation of the novel qHRP2/3-del assay. We aimed at improving the detection of $p f h r p 2$ and $p f h r p 3$ gene deletions by developing a quantitative PCR-based assay able to detect and quantify $p f h r p 2$ and $p f h r p 3$ genes in a single reaction. Given the high nucleotide sequence similarity and the repetitive structure of the $p f h r p 2$ and $p f h r p 3$ genes, nucleotide regions serving as targets for primers and probes were limited (Supplementary File 1). The primer and probe combinations selected for our assay (Table 1) bind to a region spanning exon 1 and exon 2 of both genes. Absence of amplification will therefore indicate a deletion of the entire genes or partial gene deletions including exon 1, the intron and first 96 base pairs of exon 2. Although there are chromosome breaking points outside the amplified regions, in particular the section that contains the repeats and epitopes detected by RDTs, analysis of field isolates suggest that the selected regions are highly predictive for pfhrp2/3 deletions in field strains $s^{9,12,15,16}$.

We designed a multiplex qPCR assay using three differently labelled TaqMan assays detecting the $p f h r p 2$ (PF3D7_0831800) and pfhrp3 (PF3D7_1372200) genes with the single copy gene pfrnr2e2 (PF3D7_1015800) as the internal control. The sequence alignment of the $p f h r p 2$ and $p f h r p 3$ genes highlighting the oligo binding regions is shown in Supplementary File 1.

The multiplexed assays correctly identify P. falciparum strains carrying known deletions of $p f h r p 2$ (PfDD2 strain) and $p f h r p 3$ (PfHB3 strain) as well as a strain without deletion (PfNF54 strain) (Fig. 1A). The multiplexed assays show comparable characteristics in terms of sensitivity and qPCR performance. Using DNA extracted from 
A
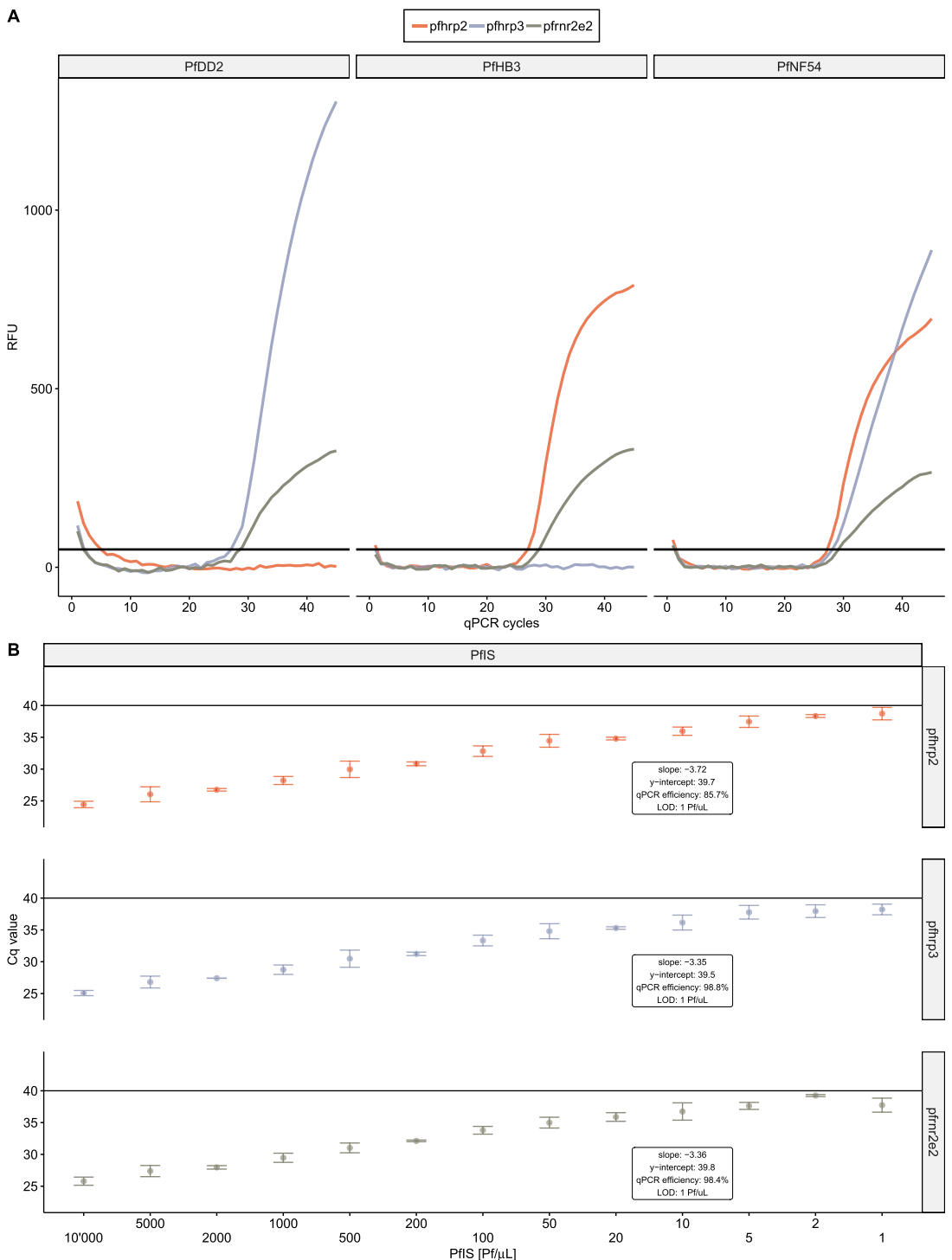

Figure 1. Multiplex detection of $p f h r p 2$ and $p f h r p 3$ genes using the qHRP2/3-del assay. (A) The qHRP2/3del assay amplifies $p f h r p 2, p f h r p 3$ and $p f r n r 2 e 2$ target sequences in a multiplex qPCR reaction and correctly identifies strains carrying either a $p f h r p 2$ deletion (PfDD2), a $p f h r p 3$ deletion (PfHB3) or no deletion (PfNF54). (B) Performance characteristic of each individual amplification assay, run within the multiplex qHRP2/3-del assay, is shown. Correlation with high linearity between serially diluted WHO international standard for $P$. falciparum NATs (PfIS) and Cq values was obtained and used to calculate the qPCR efficiency. Cq values above 40 (black line) are considered negative.

cultured parasites, all three targets are detected in samples with parasitemia as low as 1 parasite/ $\mu \mathrm{L}$ and an inverse linear correlation between Cq values and parasite densities ranging from 1 to 10 '000 parasites/ $\mu \mathrm{L}$ was observed. The qPCR efficiencies were calculated as $85.7 \%, 98.8 \%$ and $98.4 \%$ for the amplification of $p f h r p 2$, pfhrp3 and pfrnr2e2, respectively (Fig. 1B). The qHRP2/3-del assay was next tested using purified DNA from eight culture adapted P. falciparum strains from Africa (Pf3D7, PfNF54, PfNF166.C8), South and Central America (Pf7G8, PfHB3), South East Asia (PfNF135.C10, PfDD2) and Papua New Guinea (PfFC27) with known deletion status of the $p f h r p 2$ and $p f h r p 3$ genes. The Cq values for amplification of $p f r n r 2 e 2$ were comparable between the eight strains amplified and no significant differences of Cq values for the $p f h r p 2$ gene and $p f h r p 3$ gene across the strains carrying the genes was observed. Sequence alignments of PfNF135.C10, Pf3D7, Pf7G8, PfNF54 and PfNF166. C8 did not reveal sequence variation in the oligo binding regions of $p f h r p 2$ (Supplementary File 2) or $p f h r p 3$ (Supplementary File 3) supporting these findings. DNA derived from five non-falciparum Plasmodium species ( $P$. ovale curtisi, $P$. ovale wallikeri, $P$. malariae, $P$. knowlesi, $P$. vivax) was tested with the $\mathrm{qHRP} 2 / 3$-del assay and did not result in amplification of any target demonstrating the specificity for $P$. falciparum. In summary, we developed a $P$. falciparum-specific multiplex qPCR assay that allowed the simultaneous amplification of the $p f h r p 2$, $p$ fhrp 3 and $p$ frnr $2 e 2$ genes in a single reaction with high efficiency and ability to correctly identify $p f h r p 2$ and $p f h r p 3$ gene deletions. 


\begin{tabular}{|l|l|l|l|l|}
\hline Sample set & Description of sample set & $\begin{array}{l}\text { Number of } \boldsymbol{P}_{\text {. falciparum }} \\
\text { positive samples }^{\mathrm{b}}\end{array}$ & $\begin{array}{l}\text { Parasitemia in parasites/ } \\
\boldsymbol{\mu} \text { (Median/IQR) }\end{array}$ & $\begin{array}{l}\text { Amplification rate by } \\
\text { qHRP2/3-del assay }^{\mathrm{c}}\end{array}$ \\
\hline CHMI $^{\mathrm{a}}$ & CHMI in TZ with PfNF54 strain (no deletion) & 49 & $51.1(1.5-152.5)$ & $78 \%$ \\
\hline PE & $\begin{array}{l}\text { Peruvian samples around Iquitos city. High } \\
\text { proportion of } p \text { fhrp2/3 deletions }\end{array}$ & 68 & $592.4(186.7-1982.0)$ & $99 \%$ \\
\hline EG & $\begin{array}{l}\text { Blood donors with asymptomatic malaria infection } \\
\text { living on Bioko Island, Equatorial Guinea }\end{array}$ & 47 & $4.8(1.0-45.3)$ & $51 \%$ \\
\hline TZ & $\begin{array}{l}\text { Sampling of symptomatic volunteers at two health } \\
\text { facilities in Southern Tanzania }\end{array}$ & 90 & $38.8(0.7-808.6)$ & $62 \%$ \\
\hline Combined & & $\mathbf{2 5 4}$ & $\mathbf{7 5 . 7 ( 2 . 2 - 5 7 1 . 6 )}$ & $\mathbf{7 3 \%}$ \\
\hline
\end{tabular}

Table 2. Field samples used for evaluation of qHRP2/3-del assay. ${ }^{\mathrm{a} C o n t r o l l e d ~ H u m a n ~ M a l a r i a ~ I n f e c t i o n . ~}{ }^{\mathrm{b}}$ All confirmed by diagnostic qPCR assays. 'Positive for internal control of assay ( $p$ frnr $2 e 2)$.

Analysis of $P$. falciparum field strains with qHRP2/3-del assay. The qHRP2/3-del assay was next tested using a collection of 254 P. falciparum isolates originating from East Africa, Central-West Africa and Latin America (Table 2). The infection status and parasitemia levels were well established in these samples by using published diagnostic qPCR assays routinely used in the laboratories in Tanzania ${ }^{17}$, Equatorial Guinea ${ }^{18}$ and Peru $^{19}$. The overall median parasitemia in these samples was 75.7 parasites/ $\mu \mathrm{L}$ (IQR: 2.2-571.6), which is below the LOD of 100 parasites/ $\mu \mathrm{L}$ for PfHRP2-based RDTs ${ }^{20,21}$. First, the ability of the pfrnr2e2 singly copy gene to serve as internal assay control and to quantify parasitemia levels was assessed. Out of the 254 samples, 186 (73.2\%) amplified the pfrnr $2 e 2$ singly copy gene. Failure in amplification of $p f r n r 2 e 2$ was associated with low parasitemia levels (Fig. 2A). In samples with parasitemia levels of 3 parasites $/ \mu \mathrm{L}$ and above, more than $95 \%$ of all samples were amplified successfully. In samples with parasitemia $>100$ parasites $/ \mu \mathrm{L}$, the lower limit of detection for PfHRP2-based RDTs, all qPCR reactions were positive for $p f r n r 2 e 2$. Parasitemia levels determined by using the amplification of $p$ frnr2e 2 correlated closely with parasite densities obtained from P. falciparum diagnostic qPCR assays (Fig. 2B), this is supported by the findings of the Bland-Altman plot which demonstrates a high order of agreement (Fig. 2C). The average ratio of parasite quantification based on diagnostic qPCR assays and qHRP2/3-del assay is 0.8 (95\% CI: $-1.7-3.3$ ). In summary, the qHRP2/3-del assay amplifies $95 \%$ of samples with parasitemia levels of 3 parasites $/ \mu \mathrm{L}$ and above and can be used to reliably quantify parasite levels in field samples.

Identification of $p$ fhrp2 and pfhrp3 gene deletions using qHRP2/3-del assay. Next, we wanted to establish the performance of the qHRP2/3-del assay in comparison with nested PCR. Samples with known $p f h r p 2 / 3$ deletion status obtained from four different sources were included. Serial dilutions of DNA purified from PfDD2 ( $p f h r p 2$ deletion), PfHB3 ( $p f h r p 3$ deletion) and PfIS (no deletion) served as controls. Samples from CHMI using PfNF54 (no deletion) were added to test the specificity of the qHRP2/3-del assay. Two sample sets genotyped by nested PCR, one from Tanzania (TZ) dominated by P. falciparum strains without deletions and one from Peru (PE), with a high proportion of $p f h r p 2 / 3$ deletions were analysed. The Peruvian sample set consisted of 54 samples with both genes deleted and 7 samples with only one gene deleted. The qHRP2/3-del assay defines a deletion as failure of amplification of the $p f h r p 2 / 3$ genes (Fig. 3A, y axis, Cq set to 45 ) in samples which are positive for the internal control, pfrnr2e2 (Fig. 3A, $\mathrm{x}$ axis). Sensitivity is defined as the proportion of correctly identified $p f h r p 2 / 3$ deletions, while specificity is the proportion of correctly identified strains without $p f h r p 2 / 3$ deletions. All control samples with known deletion status were identified as expected (Fig. 3A, first panel). Importantly, the qHRP2/3-del assay correctly identified samples with parasitemia levels ranging from 1-10'0000 parasites/ $\mu \mathrm{L}$, demonstrating the dynamic range of at least 5 logs of this assay. In samples collected from volunteers that have undergone CHMI with PfNF54 (CHMI, $\mathrm{n}=38$ ), one sample that is positive for $p f h r p 2 / 3$ genes was wrongly detected as a double deleted parasite, resulting in a reduced specificity (Fig. 3A, second panel). A high sensitivity was achieved with the Peruvian samples (PE, $\mathrm{n}=67)$, pfhrp 2 and $p f h r p 3$ deletions were detected with sensitivity of $94.4 \%$ and $94.9 \%$, respectively (Fig. $3 \mathrm{~A}$, third panel). The low specificity of $76.9 \%$ and $87.5 \%$ for $p f h r p 2$ and $p f h r p 3$, respectively, is based on the incorrect detection of deletions in three samples. Among the samples from Tanzania (TZ, $\mathrm{n}=56$ ), no $p f h r p 2 / 3$ deletions were detected by the nested PCR. In contrast, the qHRP2/3-del assay identified three deletions, resulting in a specificity of $93.8 \%$ (Fig. 3A, fourth panel).

Grouping the samples with missed deletions (reducing the sensitivity) and the false deletions (reducing the specificity) by parasitemia levels revealed a high proportion of false deletions among the samples with the lowest parasitemia levels (Fig. 3B). Based on these findings, the inclusion criteria for samples to be analysed by qHRP2/3-del assay was changed. The threshold for the pfrnr2e2 gene amplification was reduced from $\mathrm{Cq}<40$ to $\mathrm{Cq}<37.5$, corresponding to parasitemia levels of 5 parasites $/ \mu \mathrm{L}$. Based on these new inclusion criteria, the qHRP2/3-del assay obtained results from 106 samples out of 127 samples (inclusion rate of 83.5\%) (Fig. 3C). 12 samples were not amplified by the qHRP2/3-del assay and an additional 9 samples excluded based on the new inclusion criteria. Samples which were not amplified by the qHRP2/3-del assay were mainly ultra-low parasite density samples from Tanzania (11 out of 12).

In 99 out of 106 samples (93.4\%), the pfhrp2/3 deletion status was identical when compared between qHRP2/3-del assay and nested PCR. This is reflected in the near perfect agreement between these two PCR based diagnostic methods for each of the amplified targets. Cohen's kappa was calculated as 0.89 and 0.91 for $p f h r p 2$ and $p f h r p 3$, respectively. Out of the seven samples which were misidentified in four samples both $p f h r p$ genes were affected, while in two samples the $p f h r p 2$ and in one sample the $p f h r p 3$ status was misclassified. For four misidentified samples with higher parasitemia levels the possibility of sample mix-up or cross-contamination 

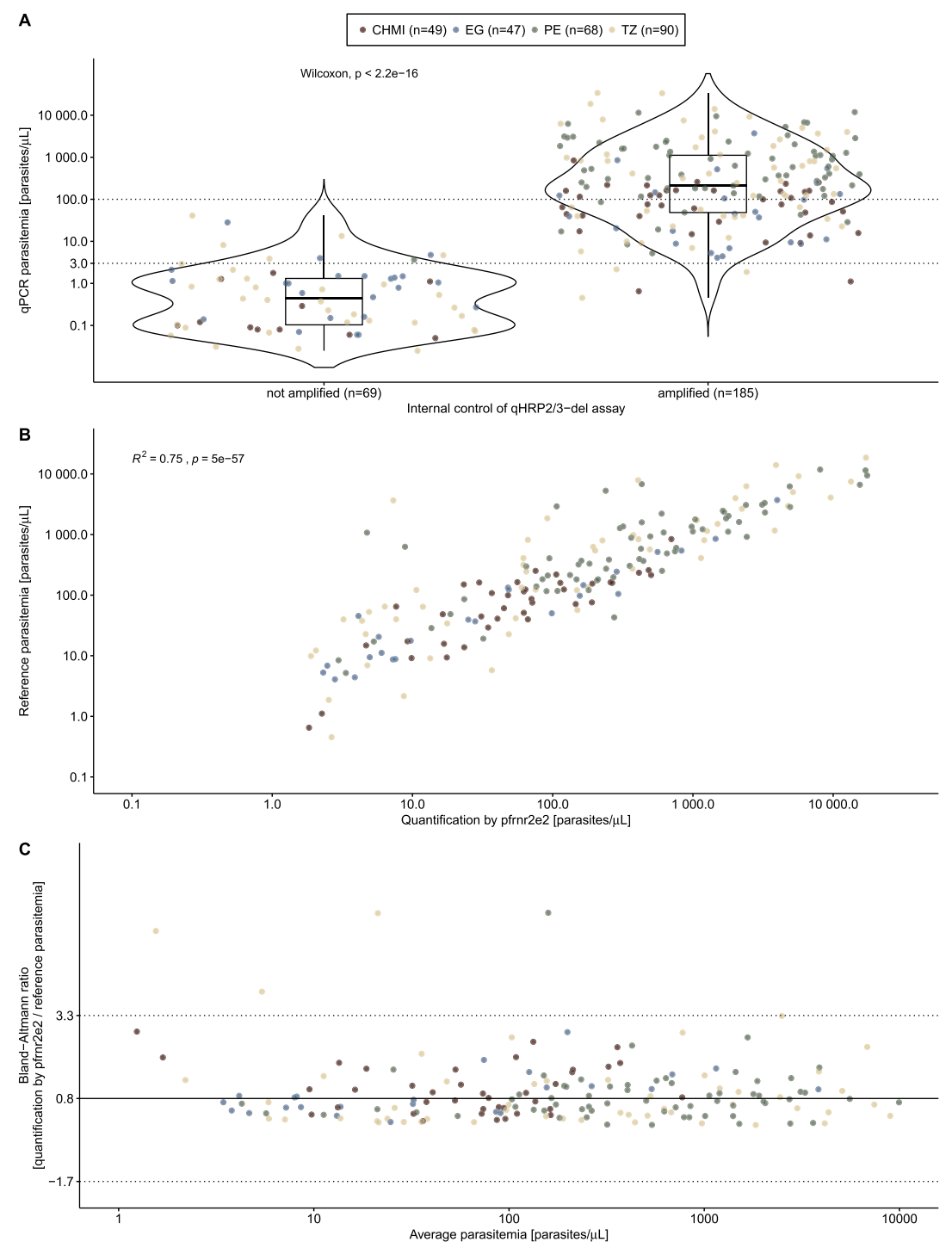

Figure 2. Detection and quantification of field samples using qHRP2/3-del assay. (A) Amplification rate of pfrnr2e 2 target, the internal control of qHRP2/3-del assay and association with parasitemia levels. WilcoxonMann-Whitney test was used for comparison of parasitemia between groups. The dashed line at 3 parasites $/ \mu \mathrm{L}$ represents the parasitemia at which more than $95 \%$ of the samples were amplified, while the dashed line at 100 parasites/ $\mu \mathrm{L}$ represents the LOD of RDTs at which all samples are successfully amplified. (B) Correlation of parasitemia levels, obtained from diagnostic qPCR assays, and parasitemia, derived from the internal control of qHRP2/3-del assay, is shown. The color represents the different sample sets and $\mathrm{R}^{2}$ the Spearman's rank correlation coefficient. (C) Bland-Altman plot of average parasitemia (x-axis) and ratio of parasitemia levels calculated between internal control of qHRP2/3-del assay and diagnostic qPCR assays ( $y$-axis). Average ratio (black line) and 95\% limits of agreement (dashed line) are depicted.

cannot be excluded, since these samples were located next to each other on the DNA plate which was shipped. In summary, the qHRP2/3-del assay specificity (94.4\% and 96.0\% for $p f h r p 2$ and $p f h r p 3$, respectively) and sensitivity ( $94.2 \%$ and $94.6 \%$ for $p f h r p 2$ and $p f h r p 3$, respectively) were above $90 \%$. The negative predictive value (NPV) was calculated as $94.5 \%$ and $94.1 \%$ and the positive predictive value (PPV) as $94.2 \%$ and $96.4 \%$, for $p f h r p 2$ and $p f h r p 3$, respectively (Fig. 3D).

Multiple strain $P$. falciparum infections are masking $p f h r p 2$ and $p f h r p 3$ deletions. In many malaria endemic regions, particularly in sub-Saharan Africa, infections with multiple strains of $P$. falciparum are common $^{22}$. A blood sample carrying multiple $P$. falciparum strains with and without $p$ fhrp $2 / 3$ deletions will result in failure to detect the deletion by nested PCR if the parasitemia level of the strain without deletion is sufficiently high for amplification. This limitation leads most likely to an underestimation of the prevalence of $p f h r p 2 / 3$ gene deletions in regions with high prevalence of multiple strain infections. We reasoned that the qHRP2/3-del assay could offer a solution by calculating the difference between the Cq values obtained for amplification of $p f h r p 2$ or $p f h r p 3$ and $p f r n r 2 e 2$. To demonstrate the ability of the qHRP2/3-del assay to correctly identify and quantify 


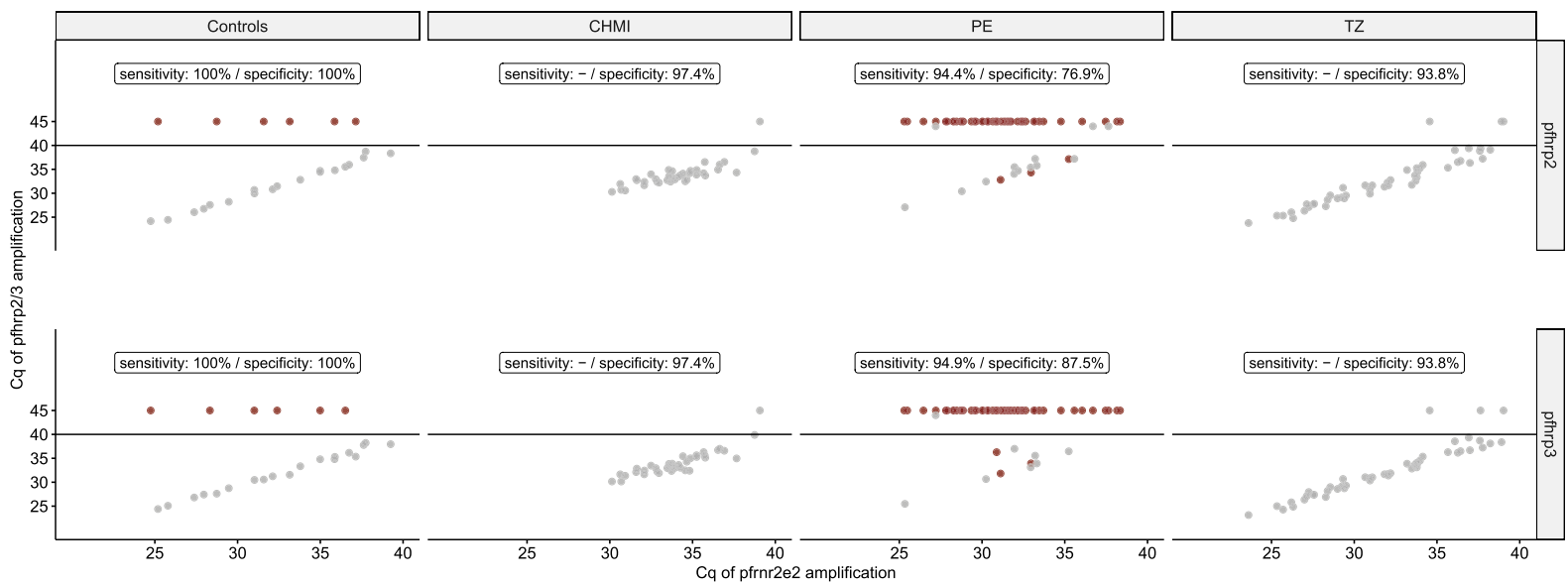

B $\quad$ qHRP2/3-del $\square$ missed deletion $\square$ false deletion $\square$ correct identification
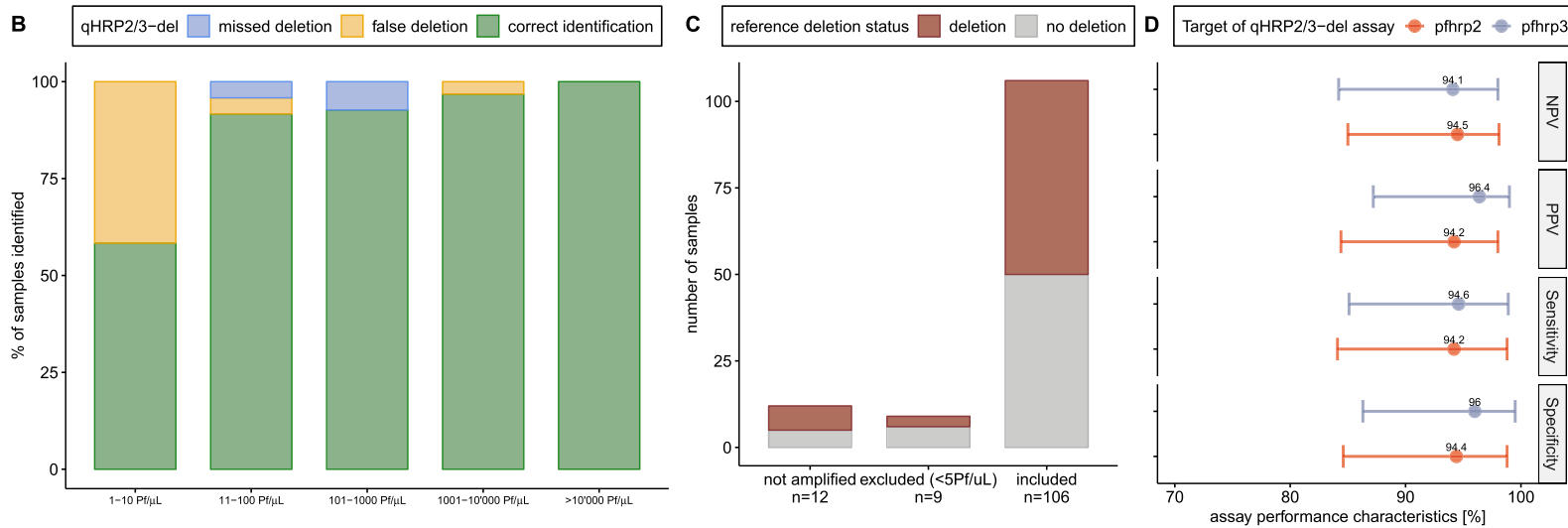

Figure 3. Diagnostic performance of qHRP2/3-del assay. (A) Samples with Cq values for $p f h r p 2$ and $p f h r p 3$ amplification $>40$ (shown on y-axis, black line indicates cut-off) are considered to carry a $p$ fhrp $2 / 3$ deletion. Reference deletion status, based on nested PCR, is color coded (red = deletion, grey $=$ no deletion). (B) Proportion of correctly and incorrectly identified $p$ fhrp 2/3 deletion status grouped by parasitemia. (C) Number of samples included for analysis by qHRP2/3-del assay $(n=106)$, excluded due to ultra-low parasitemia $(n=9)$ and not amplified $(\mathrm{n}=12)$. (D) Analytical validation of qHRP2/3-del assay performance was assessed by comparing it to nested PCR. Standard parameters such as sensitivity, specificity, positive predictive value (PPV), and negative predictive value (NPV) including their $95 \%$ confidence intervals are shown.

"hidden" or "masked" pfhrp2/3 gene deletions in mixed infections, we first tested defined mixtures of DNA from PfNF54 (no $p f h r p 2 / 3$ deletions) and PfDD2 ( $p f h r p 2$ deletion) or PfHB3 ( $p f h r p 3$ deletion) in a range of different ratios. For each combination of strain mixtures, PfDD2/PfNF54 or PfHB3/PfNF54, 10 mixtures were prepared containing varying ratios of strains with and without a $p f h r p 2 / 3$ deletion (Fig. $4 \mathrm{~A}$ ). The contribution from PfDD2 and PfHB3 strains to these mixtures ranged from $0.1 \%$ to $88 \%$ and $0.1 \%$ to $86 \%$, respectively. In seven mixtures, the strain with a deletion constituted the minority (with less than $50 \%$ abundance) and in three mixtures the majority (with more than $50 \%$ abundance). None of these mixtures failed to amplify the $p f h r p 2 / 3$ genes, even if the strain carrying the deletion constituted the majority in the mixture. A positive correlation between abundance of isolate carrying a deletion and an increase of $\Delta \mathrm{Cq}$ (Cq of $p f h r p 2$ or $p f h r p 3$ minus Cq of $p f r n r 2 e 2)$ is observed (Fig. 4B). The qHRP2/3-del assay does not only successfully identify "masked" $p f h r p 2 / 3$ deletions but can also discriminates between mixtures where the strain with the deletion constitutes the majority or minority (Fig. 4C). A $\Delta$ Cq cut-off value of 2.0 was chosen to identify "masked" pfhrp gene deletions. Applying this cut-off to our sample collections revealed that two isolates each from Tanzania and Peru have high $\Delta \mathrm{Cq}$ values for both $p f h r p$ genes indicative of the presence of "masked" pfhrp2/3 deletions (Fig. 4D). Three additional samples from the Peruvian collection had a $\Delta$ Cq value $>2$ for the $p$ fhrp 2 gene only. No $\Delta$ Cq values above 2 were found in Equatorial Guinean isolates and among samples collected from volunteers undergoing CHMI (Fig. 4D). These experiments demonstrate that by calculating the $\Delta$ Cq values between Cq for $p f r n r 2 e 2$ and $p f h r p 2$ or $p f h r p 3$, "masked" deletions can be identified.

\section{Discussion}

$P$. falciparum strains carrying $p f h r p 2 / 3$ deletions are an emerging threat to malaria control and elimination programs around the world. Novel analysis tools enabling high-throughput screening of $P$. falciparum populations from the field are needed. The currently published methods, mostly based on nested PCR, have clear limitations in that these methods are extremely time consuming, prone to detection of incorrect deletions at low parasitemia levels and unable to identify "masked" deletions in multiple strain co-infections. 

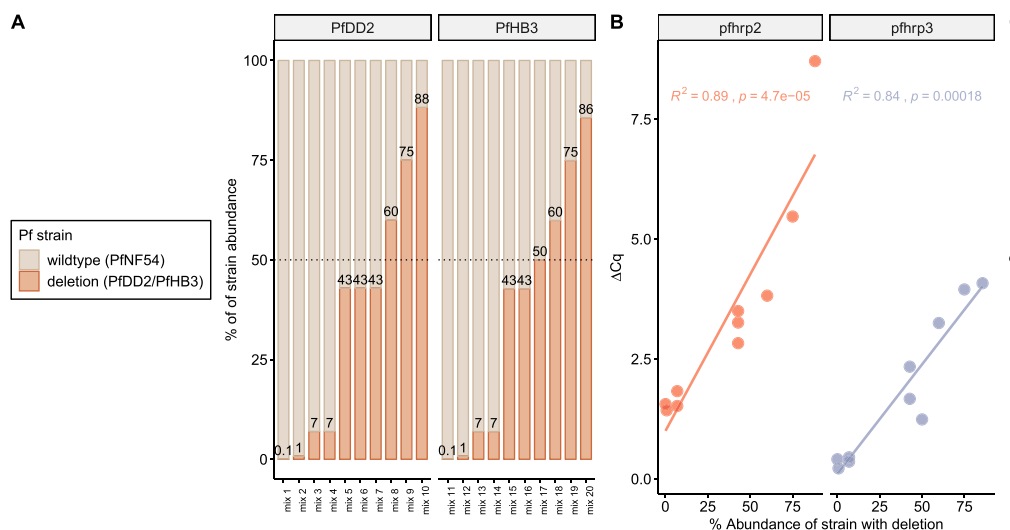

c
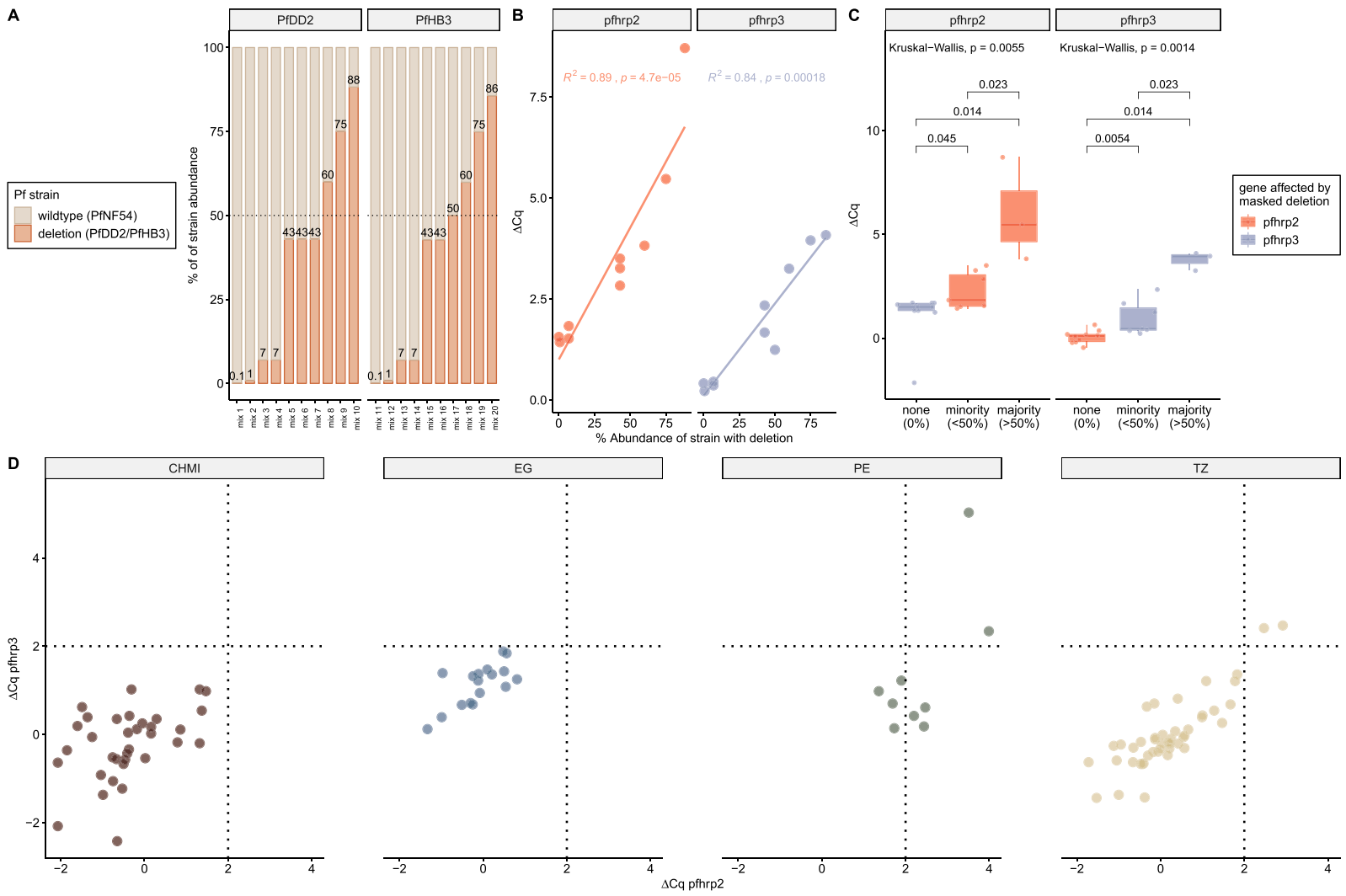

Figure 4. Identification of masked $p f h r p 2 / 3$ deletions in multiple strain infections. (A) Mixtures containing two strains, one with a $p$ fhrp deletion (PfDD2 or PfHB3) and no deletion (PfNF54), were generated. (B) Correlation between abundance of strain carrying deletion and $\Delta \mathrm{Cq}$ is shown for both targets, $p f h r p 2$ (red) and pfhrp3 (blue). (C) The $\Delta \mathrm{Cq}$ approach distinguishes between strain mixtures not carrying deletions, mixtures with minority abundance as well as majority abundance of strains with deletions. Statistical comparison was performed using the Kruskal-Wallis test followed by Wilcoxon-Mann-Whitney for pairwise comparisons. (D) The $\Delta$ Cq approach of the qHRP2/3-del assay was applied to four sample collections to identify "masked" pfhrp2/3 deletions. The control group, based on samples from CHMI, did not reveal isolates with increased $\Delta$ Cq values. The dashed lines represent the $\Delta$ Cq cut-off values for $p f h r p 2$ (x-axis) and $p f h r p 3$ (y-axis).

The sensitivity and specificity of the PfHRP2/3-del assay is comparable to the widely used nested PCR. However, the novel qHRP2/3-del assay is well suited for high throughput screening of $P$. falciparum isolates with approximately 30 samples analyzed in less than two hours - including DNA extraction and data analysis. Two additional major advantages of the qHRP2/3-del assay are obvious: firstly, the ability to quantify parasitemia levels and therefore include samples based on parasitemia and secondly, to identify "masked" deletions in multiple strain infections.

The identification of $p f h r p 2 / 3$ deletions in samples with low parasitemia levels is difficult since the absence of amplification could be due to lack of sufficient template leading to incorrect reports of deletions. The conventional method depends on the successful amplification of at least two single copy reference genes to ensure sufficient template in the PCR reaction. This procedure is time-consuming and labour-intensive. The qHRP2/3-del assay uses an alternative inclusion criterion, based on the $\mathrm{Cq}$ value of its internal control. The pre-defined exclusion criteria of all samples that have parasitemia below 5 parasites $/ \mu \mathrm{L}$ will improve the quality, reproducibility and comparability of malaria parasite survey data obtained with the qHRP2/3-del assay.

The ability to detect "masked" $p$ fhrp $2 / 3$ deletions is probably the most interesting feature of the qHRP2/3-del assay, because it will allow studying the epidemiology of $p f h r p 2 / 3$ deletions in malaria endemic regions with a high proportion of infections caused by multiplicity of infections, particular sub-Saharan Africa ${ }^{22}$. The qHRP2/3-del assay correctly identified infections that contain two strains, one with a deletion and the other one without a deletion, based on a difference in the Cq values derived from the amplification of the $p f h r p 2 / 3$ gene targets and the $p$ frnr $2 e 2$ control. However, currently we cannot exclude that nucleotide sequence variations located in the binding sites of the oligonucleotides used in the PfHRP2/3-del assay could potentially also lead to variation in $\Delta \mathrm{Cq}$ values. The $\triangle \mathrm{Cq}$ application of our novel PfHRP2/3-del assay in additional studies including a larger sample size will improve our understanding of the relevance of "masked" $p f h r p 2$ and $p f h r p 3$ gene deletions and their impact on reliability of malaria RDT test results.

Two Tanzanian isolates had an increased $\Delta$ Cq value for both $p$ fhrp genes, indicating the presence of $p f h r p 2 / 3$ deletions in the East African nation. This was recently confirmed when $p f h r p 2$ and $p f h r p 3$ deletions were 
identified in Tanzania and Uganda ${ }^{23}$. Together with findings from Kenya ${ }^{24}$, the Democratic Republic of Congo ${ }^{25}$, Rwanda $^{26}$ and Mozambique ${ }^{27}$ there is strong evidence for the existence of $p f h r p 2 / 3$ deletions in this region. Therefore, establishing programs which systematically monitor $p f h r p 2 / 3$ deletions and their impact on the performance of RDTs is advised.

\section{Conclusion}

The qHRP2/3-del assay presented here is suitable for high-throughput screening of $P$. falciparum strains to identify $p f h r p 2 / 3$ gene deletions in different malaria endemic settings, including areas with high a proportion of multiple strain co-infections. With growing availability of qPCR instruments in reference laboratories in sub-Saharan countries, this assay could be used as surveillance method to monitor over time the potential expansion of $P$. falciparum strains carrying $p$ fhrp 2 and $p f h r p 3$ deletions.

\section{Methods}

P. falciparum isolates from tanzania, equatorial guinea and peru. In this study a total of 205 $P$. falciparum isolates collected from three different malaria endemic regions, East Africa, West-Central Africa and South America were included. The samples from East Africa $(n=90)$ were collected in rural southern Tanzania (TZ) as part of a malaria baseline surve ${ }^{28}$. The West-Central African isolates $(n=47)$ were identified among blood donors living in Malabo, Equatorial-Guinea $(\mathrm{EG})^{18}$. Both samples sets were analyzed locally, at the Bagamoyo branch of the Ifakara Health Institute and the laboratory of the Equatorial Guinea Malaria Vaccine Initiative using harmonized protocols. Briefly, genomic DNA was isolated either from 6 circles with $2 \mathrm{~mm}$ diameter of dried blood spots (Tanzania) or $180 \mu \mathrm{L}$ whole blood (Equatorial Guinea) using the Quick-DNA Miniprep kits (Zymo Research, Irvine, USA). P. falciparum was identified and quantified using published qPCR protocols based on varATS ${ }^{29}$. Extracted DNA $(n=68)$ from Peruvian isolates (PE), collected between 2008-2009 and 2015-2016 around Iquitos city, was shipped to the Swiss Tropical and Public Health Institute for $p f h r p 2 / 3$ characterization by qHRP2/3-del assay.

Additional parasite isolates and laboratory strains. Forty-nine PfNF54 isolates from Controlled Human Malaria Infections (CHMI) conducted in Bagamoyo, Tanzania (ClinicalTrials.gov: NCT02613520 ${ }^{30}$ ) as well as genomic DNA isolated from 8 laboratory strains with known $p f h r p 2 / 3$ deletion status (Pf3D7, Pf7G8, PfDD2, PfHB3, PfNF135.C10, PfNF166.C8, PfNF54 and PfFC27) were used as controls. The 1st WHO International Standard for Plasmodium falciparum DNA Nucleic Acid Amplification Techniques (NIBSC code: 04/176, herein referred to as PfIS) was used to assess the performance of the qHRP2/3-del assay. Non-falciparum Plasmodium species, including P. malariae (Pm), P. ovale curtisi (Poc), P. ovale wallikeri (Pow), P. vivax (Pv) and $P$. knowelsi $(P k)$ and an additional 28 samples from malaria negative individuals living in Tanzania were used to assess specificity of the assay.

Detection of the $p f h r p 2$ and $p$ fhrp3 genes by conventional nested PCR. $\quad P$. falciparum positive samples collected in Tanzania were selected for detection of $p f h r p 2$ and $p f h r p 3$ genes by nested PCR. As a reference gene, the $m s p 2$ gene was amplified using a previously described protocol ${ }^{31}$. All isolates with successful $m s p 2$ amplification were analyzed for the presence of $p f h r p 2$ and $p f h r p 3$ genes using primers spanning exon 1 , the intron, and exon $2^{32}$. All PCR products were separated and visualized on a $2 \%$ agarose gel. Cultured parasite isolate PfDD2 ( $p f h r p 2$ deletion) was used as a control for all nested PCR experiments on $p f h r p 2$ while PfHB3 ( $p f h r p 3$ deletion) was used as a control for all nested PCR experiments on $p f h r p 3$. PfNF54 (no $p$ fhrp2/3 deletion) was used as a positive control for both $p$ fhrp genes. Pfhrp $2 / 3$ deletion status of the Peruvian $P$. falciparum isolates was analyzed previously following the procedures described elsewhere ${ }^{9}$. Results were shared to be used for the evaluation of the qHRP2/3-del assay.

Design of qHRP2/3-del assay. Published $p f h r p 2 / 3$ primer sequences for conventional PCR were adapted to the qPCR platform using EvaGreen ${ }^{\circledR}$ qPCR Mix (Solis BioDyne, Tartu, Estonia). The primers were tested with different DNA concentrations extracted from PfNF54, PfDD2 and PfHB3 strains, corresponding to parasitemia levels of 1 and 100 parasites/ $\mu \mathrm{L}$. The best performing primer pairs, in terms of specificity and sensitivity, were then used in combination with newly designed TaqMan ${ }^{\circledR}$ hydrolysis probes. The $p f h r p 2 / 3$ oligo sequences were systematically optimized using the trial-and-error approach. As the internal control of the qHRP2/3-del assay we amplify a $P$. falciparum specific 107 bp long sequence of the ribonucleotide reductase R2_e2 (pfrnr2e2), a distantly related paralog of the canonical eukaryotic small subunit ribonucleotide reductase R2, that is unique to apicomplexan species ${ }^{14}$. The performance of $p f r n r 2 e 2$ as a biomarker for detection and quantification of $P$. falciparum was tested by direct comparison with parasitemia levels obtained from a $18 \mathrm{~S}$ rDNA based qPCR assay ${ }^{33}$. A sensitivity of $89.1 \%$ for samples with parasitemia $>1$ parasite/ $\mu \mathrm{L}$ and a Bland-Altman ratio of 0.99 (95\% CI: $-0.012-$ 2.5) demonstrate its robustness and accuracy as internal control (Supplementary File 4). Genomic sequences for pfrnr2e2 (PF3D7_1015800), pfhrp2 (PF3D7_0831800) and pfhrp3 (PF3D7_1372200) of Pf3D7 strain were obtained from PlasmoDB. A pfhrp $2 / 3$ sequence alignment including five reference strains from West-Africa (Pf3D7, PfNF54), Guinea (PfNF166.C8), Brazil (Pf7G8) and Cambodia (PfNF135.C10) revealed no SNPs in oligo binding regions suggesting a high degree of conservation within the target region of the $p f h r p 2 / 3$ genes (Supplementary Files 2 and 3). The $p f h r p 2$, $p f h r p 3$ and $p f r n r 2 e 2$ sequences for Pf7G8, PfNF135.C10, PfNF166.C8 and PfNF54 were obtained from whole genome sequencing ${ }^{34}$. The Geneious version 8.1.9 software (Biomatters Ltd, Auckland, New Zealand) was used for sequence alignments and oligo designs. Relevant information concerning the oligos used in the qHRP2/3-del assay is summarized in Table 1.

Sample analysis with qHRP2/3-del assay. Amplification and qPCR measurements were performed using the Bio-Rad CFX96 Real-Time PCR System (Bio-Rad Laboratories, California, USA). The thermal profile used for qHRP2/3-del assay is as follows: Taq polymerase activation for $5 \mathrm{~min}$ at $95^{\circ} \mathrm{C}$, followed by 45 cycles of 
$15 \mathrm{~s}$ at $95^{\circ} \mathrm{C}$ and $35 \mathrm{~s}$ at $57.5^{\circ} \mathrm{C} .2 \mu \mathrm{L}$ DNA was added to $8 \mu \mathrm{L}$ reaction master mix containing $1 \mathrm{x}$ Luna Universal Probe qPCR Master Mix (New England Biolabs, Ipswich, USA) and 1x qHRP2/3-del Primer Mix (Table 1). All qPCR assays were run in triplicates with appropriate controls including Non-Template Control and DNA from PfDD2, PfHB3 and PfNF54 as controls for the $p f h r p 2 / 3$ deletion status.

Data management and statistical analysis. Preliminary analysis of qPCR data. Cq values were obtained from the Bio-Rad CFX96 Manager 3.1 software (Bio-Rad Laboratories, California, USA) after setting the threshold manually. Cq values were transferred and linked to the samples' metadata using a custom-designed database for storage and analysis of qPCR data. Only samples with a $\mathrm{Cq} \leq 40.0$ for the internal control, pfrnr2e2, were considered eligible for analysis of $p f h r p 2 / 3$ deletion status. $\Delta$ Cq were calculated by subtraction of $p f r n r 2 e 2$ Cq values from $p f h r p 2$ or $p f h r p 3 \mathrm{Cq}$ values.

Analytical performance of $q H R P 2 / 3-$ del assay and quantification of parasitemia. Based on the PfIS a serial dilution ranging from $0.01-10$ '000 parasites/ $\mu \mathrm{L}$ was prepared and used to assess the performance of the qHRP2/3-del assay. The slope, $y$-intercept, qPCR efficiency and $\mathrm{R}^{2}$ was established for each target. The Limit of Detection (LOD) was defined as the lowest PfIS parasitemia with a positive amplification in 4 out of 6 replicates. Parasitemia was estimated using linear regression derived from serial dilution of the PfIS and the $p f r n r 2 e 2$ target which serves as the internal control of the $\mathrm{qHRP} 2 / 3$-del assay.

Graphical representation and statistical analysis. We used R version 3.5.1 for creating ggplot2-based graphs using the packages ggpubr, gridextra and scales. The Diagnostic test evaluation calculator (freely available at https:// www.medcalc.org/calc/diagnostic_test.php) was used for analytical validation of qHRP2/3-del assay performance. Cohen's kappa including $95 \%$ confidence intervals, providing a measure of agreement, was calculated using STATA version 12.0 software (Stata Corp LP; College Station, Texas, USA). P values $<0.05$ were considered as significant for all statistical analysis.

Ethical approval and informed consent. The samples analyzed in this study were collected in different studies. All studies were approved by the appropriate institutions and informed consent was obtained from all participants. The CHMI samples were collected during a clinical study, registered at Clinical Trials.gov (NCT02613520), and conducted under a U.S. FDA IND application. The study was performed in accordance with Good Clinical Practices. All samples analyzed in this publication were obtained according to the approved study protocol. The protocol was approved by the institutional review boards of the Ifakara Health Institute (IHI/IRB/ No: 32-2015), and the National Institute for Medical Research Tanzania (NIMR) (NIMR/HQ/R.8a/Vol.IX/2049), by the Ethikkommission Nordwest- und Zentralschweiz, Basel, Switzerland (Ref. No. 15/104), and by the Tanzania Food and Drug Authority (Auth. No. TZ15CT013). For the Tanzanian sample collection ethics approval for the study was granted by the institutional review boards of Ifakara Health Institute (IHI/IRB/No: 18-2015) and by NIMR (NIMR/HQ/R.8a/Vol.IX/2015). For the sample collection from Equatorial Guinea approval was given by the Ministry of Health and Social Welfare. The collection, transport and storage of the blood samples from Peru was approved by the Human Ethics Committee from Universidad Peruana Cayetano Heredia (UPCH 52707 \& 59751).

\section{Data Availability}

All data generated or analyzed during this study are included in this published article and its Supplementary Information Files.

\section{References}

1. WHO. World malaria report 2018. World Heal. Organ. 210 (2019).

2. Wongsrichanalai, C., Barcus, M. J., Muth, S., Sutamihardja, A. \& Wernsdorfer, W. H. A review of malaria diagnostic tools: microscopy and rapid diagnostic test (RDT). Am J Trop Med Hyg 77 (2007).

3. Boyce, M. R. \& O'Meara, W. P. Use of malaria RDTs in various health contexts across sub-Saharan Africa: a systematic review. BMC Public Health 17 (2017).

4. Mouatcho, J. C. \& Dean Goldring, J. P. Malaria rapid diagnostic tests: Challenges and prospects. J. Med. Microbiol. 62, 1491-1505 (2013).

5. Howard, R. J. et al. Secretion of a malarial histidine-rich protein (Pf HRP II) from Plasmodium falciparum-infected erythrocytes. J. Cell Biol. 103, 1269-1277 (1986).

6. Baker, J. et al. Global sequence variation in the histidine-rich proteins 2 and 3 of Plasmodium falciparum: Implications for the performance of malaria rapid diagnostic tests. Malar. J. 9, 1-12 (2010).

7. Bousema, T., Okell, L., Felger, I. \& Drakeley, C. Asymptomatic malaria infections: detectability, transmissibility and public health relevance. Nature reviews. Microbiology 12, 833-840 (2014).

8. Baker, J. et al. Transcription and expression of Plasmodium falciparum Histidine-Rich proteins in different stages and strains: Implications for rapid diagnostic tests. PLoS One 6 (2011).

9. Gamboa, D. et al. A Large Proportion of P. falciparum Isolates in the Amazon Region of Peru Lack pfhrp2 and pfhrp3: Implications for Malaria Rapid Diagnostic Tests. PLoS One 5, e8091 (2010).

10. Gendrot, M., Fawaz, R., Dormoi, J., Madamet, M. \& Pradines, B. Genetic diversity and deletion of Plasmodium falciparum histidinerich protein 2 and 3: a threat to diagnosis of P. falciparum malaria. Clin. Microbiol. Infect. 2-7, https://doi.org/10.1016/j. cmi.2018.09.009 (2018).

11. Verma, A. K., Bharti, P. K. \& Das, A. HRP-2 deletion: a hole in the ship of malaria elimination. Lancet. Infect. Dis. 18, 826-827 (2018).

12. Cheng, Q. et al. Plasmodium falciparum parasites lacking histidine-rich protein 2 and 3: a review and recommendations for accurate reporting. Malar J 13, 283 (2014).

13. Sepúlveda, N. et al. Global analysis of Plasmodium falciparum histidine-rich protein-2 (pfhrp2) and pfhrp3 gene deletions using whole-genome sequencing data and meta-analysis. Infect. Genet. Evol. 62, 211-219 (2018).

14. Munro, J. B., Jacob, C. G. \& Silva, J. C. A novel clade of unique eukaryotic ribonucleotide reductase R2 subunits is exclusive to apicomplexan parasites. J. Mol. Evol. 77, 92-106 (2013).

15. Fontecha, G. et al. Deletions of pfhrp2 and pfhrp3 genes of Plasmodium falciparum from Honduras, Guatemala and Nicaragua. Malar. J. 17, 320 (2018). 
16. Dharia, N. V. et al. Genome scanning of Amazonian Plasmodium falciparum shows subtelomeric instability and clindamycinresistant parasites. Genome Res. 20, 1534-44 (2010).

17. Jongo, S. A. et al. Safety and Differential Antibody and T-Cell Responses to Plasmodium falciparum Sporozoite Vaccine by Age in Tanzanian Adults, Adolescents, Children, and Infants. Am. J. Trop. Med. Hyg., https://doi.org/10.4269/ajtmh.18-0835 (2019).

18. Schindler, T. et al. Molecular monitoring of the diversity of human pathogenic malaria species in blood donations on Bioko Island, Equatorial Guinea. Malar. J. 18, 9 (2019).

19. Serra-Casas, E. et al. Loop-mediated isothermal DNA amplification for asymptomatic malaria detection in challenging field settings: Technical performance and pilot implementation in the Peruvian Amazon. PLoS One 12, 1-19 (2017).

20. Ochola, L. B., Vounatsou, P., Smith, T., Mabaso, M. L. H. \& Newton, C. R. J. C. The reliability of diagnostic techniques in the diagnosis and management of malaria in the absence of a gold standard. Lancet. Infect. Dis. 6, 582-588 (2006).

21. Bell, D., Wongsrichanalai, C. \& Barnwell, J. W. Ensuring quality and access for malaria diagnosis: how can it be achieved? Nat. Rev. Microbiol. 4, S7-20 (2006).

22. Balmer, O. \& Tanner, M. Prevalence and implications of multiple-strain infections. Lancet Infect. Dis. 11, 868-878 (2011).

23. Thomson, R. et al. pfhrp2 and pfhrp3 gene deletions that affect malaria rapid diagnostic tests for Plasmodium falciparum: analysis of archived blood samples from three African countries. J. Infect. Dis., https://doi.org/10.1093/infdis/jiz335 (2019).

24. Beshir, K. B. et al. Plasmodium falciparum parasites with histidine-rich protein 2 (pfhrp2) and pfhrp3 gene deletions in two endemic regions of Kenya. Sci. Rep. 7, 14718 (2017).

25. Parr, J. B. et al. Pfhrp2-deleted Plasmodium falciparum parasites in the Democratic Republic of the Congo: a national cross-sectional survey. J. Infect. Dis. 216, 36-44 (2016).

26. Kozycki, C. T. et al. False-negative malaria rapid diagnostic tests in Rwanda: impact of Plasmodium falciparum isolates lacking hrp2 and declining malaria transmission. Malar. J. 16, 123 (2017).

27. Gupta, H. et al. Molecular surveillance of pfhrp2 and pfhrp3 deletions in Plasmodium falciparum isolates from Mozambique. Malar. J. 16, 416 (2017).

28. Khatib, R. A. et al. Epidemiological characterization of malaria in rural southern Tanzania following China-Tanzania pilot joint malaria control baseline survey. Malar. J. 17, 292 (2018).

29. Hofmann, N. et al. Ultra-Sensitive Detection of Plasmodium falciparum by Amplification of Multi-Copy Subtelomeric Targets. PLOS Med. 12, e1001788 (2015).

30. Jongo, S. A. et al. Safety and differential antibody and T cell responses to PfSPZ Vaccine by age in Tanzanian adults, adolescents, children and infants. Am. J. Trop. Med. Hyg. In press (2019).

31. Atroosh, W. M. et al. Genetic diversity of Plasmodium falciparum isolates from Pahang, Malaysia based on MSP-1 and MSP-2 genes. Parasit. Vectors 4, 233 (2011).

32. Abdallah, J. F. et al. Prevalence of pfhrp2 and pfhrp3 gene deletions in Puerto Lempira, Honduras. Malar. J. 14, 19 (2015).

33. Kamau, E., Alemayehu, S., Feghali, K. C., Saunders, D. \& Ockenhouse, C. F. Multiplex qPCR for Detection and Absolute Quantification of Malaria. PLoS One 8 (2013).

34. Moser, K. A. et al. Strains used in whole organism Plasmodium falciparum vaccine trials differ in genome structure, sequence, and immunogenic potential. bioRxiv 684175, https://doi.org/10.1101/684175 (2019).

\section{Acknowledgements}

This study was funded by a public-private partnership, the Equatorial Guinea Malaria Vaccine Initiative (EGMVI), consisting of the Government of Equatorial Guinea - Ministry of Mines and Energy and Ministry of Health and Social Welfare, Marathon EG Production Limited, Noble Energy and Atlantic Methanol Production Company. We would like to thank Paul Lansdell, PHE Malaria Reference Laboratory and Don van Schalkwyk, Dept. of Immunology \& Infection, London School of Hygiene \& Tropical Medicine who kindly provided the DNA controls for non-falciparum malaria species. We also thank Matthew Adams, Institute for Global Health, University of Maryland, Robert W. Sauerwein, Radboud Center for Infectious Diseases, Radboud University Medical Center, and Chris J. Janse, Department of Parasitology, Leiden University Medical Centre, for sharing the DNA of the of $P$. falciparum strains PfNF166.C8, PfNF135.C10 and Pf7G8. Finally, we would like to thank Christin Gumpp, Christian Scheurer and Sergio Wittlin, Swiss Tropical and Public Health Institute, for their help with cultivating PfNF54, PfDD2 and PfHB3 parasites. Peruvian sample collection and storage was supported by US Public Health Service Grants U19AI089681.

\section{Author Contributions}

Study concept and design: T.S., A.C.D., C.D. Acquisition of data: T.S., A.C.D., M.F., E.G., S.M.M. Analyses and interpretation of data: T.S., A.C.D., D.G., P.M.V., C.D. Drafting the manuscript: T.S., A.C.D., C.D. Technical or material support: K.M., J.C.S., D.G., P.M.V., K.T., S.A., S.L.H., M.T. Sample collection and enrollment of patients: M.G.M., S.A.J., P.P.C., J.R.B., P.M., K.T. Study supervision: C.D. All authors read and approved the final manuscript.

\section{Additional Information}

Supplementary information accompanies this paper at https://doi.org/10.1038/s41598-019-49389-2.

Competing Interests: The authors declare no competing interests.

Publisher's note: Springer Nature remains neutral with regard to jurisdictional claims in published maps and institutional affiliations.

Open Access This article is licensed under a Creative Commons Attribution 4.0 International License, which permits use, sharing, adaptation, distribution and reproduction in any medium or format, as long as you give appropriate credit to the original author(s) and the source, provide a link to the Creative Commons license, and indicate if changes were made. The images or other third party material in this article are included in the article's Creative Commons license, unless indicated otherwise in a credit line to the material. If material is not included in the article's Creative Commons license and your intended use is not permitted by statutory regulation or exceeds the permitted use, you will need to obtain permission directly from the copyright holder. To view a copy of this license, visit http://creativecommons.org/licenses/by/4.0/.

(c) The Author(s) 2019 